\title{
Transcriptome profiling of dorsal root ganglia in a rat model of complex regional pain syndrome type-I reveals potential mechanisms involved in pain
}

This article was published in the following Dove Press journal:

Journal of Pain Research

\author{
Chengyu Yin ${ }^{1,2, *}$ \\ Qimiao $\mathrm{Hu}^{\mathrm{I}, *}$ \\ Boyu Liu' ${ }^{1} *$ \\ Yan Tai ${ }^{3}$ \\ Xiaoli Zheng' \\ Yuanyuan $\mathrm{Li}^{1}$ \\ Xuaner Xiang' \\ Ping Wang ${ }^{4}$ \\ Boyi Liu' \\ 'Department of Neurobiology and \\ Acupuncture Research, The Third \\ Clinical Medical College, Zhejiang \\ Chinese Medical University, Key \\ Laboratory of Acupuncture and \\ Neurology of Zhejiang Province, \\ Hangzhou, People's Republic of China; \\ ${ }^{2}$ College of Life Science, Zhejiang \\ Chinese Medical University, Hangzhou, \\ People's Republic of China; ${ }^{3}$ Academy of \\ Chinese Medicine Sciences, Zhejiang \\ Chinese Medical University, Hangzhou, \\ People's Republic of China; ${ }^{4}$ Department \\ of Pathology, School of Basic Medical \\ Science, Zhejiang Chinese Medical \\ University, Hangzhou, People's Republic \\ of China
}

*These authors contributed equally to this work

Correspondence: Ping Wang

Department of Pathology, School of Basic Medical Science, Zhejiang Chinese Medical University, 548 Binwen Road, Hangzhou

3 I0053, People's Republic of China

Email wangping897@I63.com

Boyi Liu

Department of Neurobiology and

Acupuncture Research, The Third Clinical

Medical College, Zhejiang Chinese Medical

University, 548 Binwen Road, Hangzhou

310053, People's Republic of China

Email boyi.liu@foxmail.com
Purpose: Complex regional pain syndrome type-I (CRPS-I) is a progressive and devastating pain condition, which remains clinically challenging. The mechanisms of CRPS-I still remain largely unknown. We aim to identify transcriptome profiles of genes relevant to pain mechanisms and major pathways involved in CRPS-I.

Methods: A rat model of chronic post-ischemia pain (CPIP) was established to mimic CRPS-I. RNA-sequencing (RNA-Seq) was used to profile transcriptome of L4-6 dorsal root ganglia (DRGs) of a rat model of CRPS-I.

Results: CPIP model rats developed persistent mechanical/thermal hyperalgesia in ipsilateral hind paw. RNA-Seq identified a total of 295 differentially expressed genes (DEGs), including 195 upand 100 downregulated, in ipsilateral DRGs of CPIP rats compared with sham rats. The expression of several representative genes was confirmed by qPCR. Functional analysis of DEGs revealed that the most significant enriched biological processes of upregulated genes include response to lipopolysaccharide, inflammatory response and cytokine activity, which are all important mechanisms mediating pain. We further screened DEGs implicated in pain progress, genes enriched in small- to medium-sized sensory neurons and enriched in TRPV1-lineage nociceptors. By comparing our dataset with other published datasets of neuropathic or inflammatory pain models, we identified a core set of genes and pathways that extensively participate in CPIP and other neuropathic pain states.

Conclusion: Our study identified transcriptome gene changes in DRGs of an animal model of CRPS-I and could provide insights into identifying promising genes or pathways that can be potentially targeted to ameliorate CRPS-I.

Keywords: RNA-Seq, pain, CRPS-I, dorsal root ganglion, neuropathic pain

\section{Introduction}

Complex regional pain syndrome type-I (CRPS-I) is a progressive and devastating pain condition that can be initiated by surgery, ischemia or fractures to the extremities. ${ }^{5,15}$ CRPS-I is usually induced after an initial noxious stimulus and followed with edema, skin blood flow changes, thermal and mechanical hyperalgesia or allodynia in affected body parts. CRPS-I can develop into chronic pain condition that dramatically affects the patient's life quality. ${ }^{49}$ Current available treatment options, such as nonsteroidal antiinflammatory drugs, corticosteroids and physiotherapy, etc cannot produce satisfactory relieving effects on CRPS-I, rendering it a clinically difficult to treat pain condition. ${ }^{27}$ 
At present, the mechanisms underlying CRPS-I are still not fully elucidated. Ischemia/reperfusion injury is among one of the causes of CRPS-I. ${ }^{4,15}$ In order to gain more insights into the molecular mechanisms of CRPS-I, Coderre et al established a rat chronic postischemic pain (CPIP) model by applying prolonged hind paw ischemia and reperfusion to mimic CRPS-I. ${ }^{16}$ The CPIP model manifests many key CRPS-I-like symptoms, including early swelling, hyperemia, hind paw warmth, and longlasting neuropathic pain condition. ${ }^{16,35,39}$ Therefore, the CPIP model is a frequently used animal model for mechanistic study of CRPS-I.

Recently, the advances in RNA-sequencing (RNA-Seq) technique and bioinformatics have made it possible to perform whole transcriptome analysis of gene changes. RNA-Seq technique has been used to profile transcriptome of sensory ganglia in physiological conditions as well as to explore gene changes under inflammatory or neuropathic pain conditions. ${ }^{2,22,24,28}$ The dorsal root ganglion (DRG) contains primary sensory neurons, which are responsible for receiving pain signals in response to noxious stimulation and conveying pain signals to spinal cord dorsal horn and play an important role in pain signal integration, transduction and peripheral sensitization. ${ }^{3}$ In order to investigate the molecular mechanisms of CRPS-I-induced pain and explore possible therapeutic options, we performed transcriptome analysis of the DRGs that innervate the affected hind limb of CPIP model rats. We determined the overall profiles of differentially expressed genes (DEGs) in CPIP model and screened the genes that are possibly involved in pain mechanisms and nociception. We further investigated the major pathways or functions that these DEGs are possibly involved in. Finally, we compared our findings with previously published microarray datasets of neuropathic and inflammatory pain models and we identified a core set of genes and pathways that extensively participate in CPIP and other neuropathic pain states. Therefore, our study will further advance our understanding of the peripheral neuronal mechanisms of CRPSI-induced pain symptom and help to identify promising genes that can be targeted for CRPS-I treatment.

\section{Material and methods}

\section{Animals}

Male Sprague Dawley rats (from Laboratory of Animal Research Center, Zhejiang Chinese Medical University, Hangzhou, China), weighting 300-320 g, were used in this study. The rats were housed five per cage on a 12 hour light/dark cycle with controlled temperature. Food and water were provided ad libitum. The rats were given minimum of 1 week to adapt to the new environment before experiment. All experimental procedures were approved by the Animal Ethics Committee of Zhejiang Chinese Medical University and carried out according to the National Institutes of Health guide for the care and use of Laboratory animals (NIH Publications No. 8023, revised 1978).

\section{CPIP rat model establishment}

CPIP was established through exposure to prolonged hind paw ischemia and reperfusion as described previously. ${ }^{16}$ Anesthesia was induced in all rats with an intraperitoneal injection of $50 \mathrm{mg} / \mathrm{kg}$ of sodium phenobarbital and was maintained with an infusion of sodium phenobarbital at 20 $\mathrm{mg} / \mathrm{kg}$ /hour. An O-ring with 7/32 internal diameter was tightly passed around the right hind limb just proximal to the ankle joint. The O-ring was then cut off 3 hours later for reperfusion. Sham rats received the same anesthetic procedure but the ankle was surrounded with a cut O-ring which did not block blood flow.

\section{Behavioral test}

Rats were habituated to the test environment daily for a consecutive 3 days before baseline test. Rats were individually placed in transparent plexiglas chambers on an elevated mesh floor and were habituated for 30 minutes before test. The mechanical allodynia was determined using a series of von Frey filaments (UGO Basile, Italy) applied perpendicularly to the mid plantar surface of the hind paws, with sufficient force to bend the filament slightly for 3-5 seconds according to methods previously described. ${ }^{9}$ An abrupt withdrawal of the paw and licking and vigorously shaking in response to stimulation were considered pain-like responses. The threshold was determined using the up-down testing paradigm, and the 50\% paw withdrawal threshold was calculated by the nonparametric Dixon test. ${ }^{29,30}$

The Plantar Test Apparatus (Ugo Basile) was used to evaluate thermal hyperalgesia according to methods previously described. ${ }^{7}$ A radiant light beam generated by a light bulb was directed into the right hind paw in order to determine the paw withdrawal latency (the time spent to remove the paw from the stimulus). A 25 second cutoff threshold was set to avoid excessive heating to cause 
injury. Significant decreases in paw withdrawal latency were interpreted as thermal hyperalgesia. All the above behavior tests are conducted by an experimenter blinded to experimental conditions.

\section{Tissue collect and RNA extraction}

At day 7, rats were deeply anesthetized with sodium pentobarbital $(40 \mathrm{mg} / \mathrm{kg}$ ) and perfused through the ascending aorta with saline. After the perfusion, the L4-6 DRG segments were collected. Total RNA from the CPIP and sham groups was extracted using TRIzol reagent (Thermo Fisher Scientific, Waltham, MA, USA) following the manufacturer's instructions, and then treated with DNase I to degrade contaminating DNA. The purity and concentration of the samples was evaluated using Nanodrop spectrophotometer (NanoDrop Products, CA, USA). The RNA integrity number was measured using an Agilent 2100 BioAnalyzer (Agilent Technologies, Santa Clara, CA, USA).

\section{RNA-Seq library establishment and RNA-Seq}

Total mRNAs from three rats of from CPIP group and three rats from sham groups were isolated.

DNase I was used to degrade double-stranded and singlestranded DNA contaminant in RNA samples. mRNA molecules were purified from total RNA using oligo(dT)-attached magnetic beads. mRNA were fragmented into small pieces using fragmentation reagent. First-strand cDNA was generated using random hexamer-primed reverse transcription, followed by a second-strand cDNA synthesis. The synthesized cDNA was subjected to end-repair and then was $3^{\prime}$ adenylated. Adaptors were ligated to the ends of these $3^{\prime}$ adenylated cDNA fragments. This process is to amplify the cDNA fragments with adaptors from previous step. PCR products are purified with the SPRI beads, and dissolved in EB solution. The double stranded PCR products were heat denatured and circularized by the splint oligo sequence. The single-strand circle DNA (ssCir DNA) was formatted as the final library. Library was validating on the Agilent Technologies 2100 bioanalyzer. The library was amplified with phi29 to make DNA nanoball (DNB) which have more than 300 copies of one molecular. The DNBs were loaded into the patterned nanoarray and single end 50 base reads were generated in the way of sequenced by synthesis. Finally, the fragments were enriched by PCR amplification to construct a library ready for sequencing using BGISEQ- 500 by
BGI Group (Shenzhen, China). The raw data of gene expression changes is provided as Table S1.

\section{Bioinformatics analysis}

Primary sequencing data produced by RNA-Seq (raw reads) were subjected to quality control (QC). Raw reads were filtered into clean reads by internal software SOAPnuke (version 1.5.2), followed by: Remove reads in which unknown bases $(\mathrm{N})$ are more than 10\%; Remove reads with adaptors; Remove low quality reads (we define the low quality read as the percentage of base which quality is less than 15 and greater than $50 \%$ in a read). QC of alignment was performed to determine if re-sequencing was needed. If the alignment result passed QC, downstream analysis including gene expression, differentially expressed genes, cluster analysis, gene ontology (GO) enrichment analysis, KEGG (Kyoto Encyclopedia of Genes and Genomes) pathway enrichment analysis, etc proceeded.

\section{Cluster analysis and screening of differentially expressed genes}

Distances of expressed genes were calculated using the Euclidean method. ${ }^{12}$ The sum of the squared deviations algorithm was used to calculate distance. The cluster analysis and heat map visualization of gene expression patterns was performed using "pheatmap" package in the $\mathrm{R}$ software of Bioconductor. Differentially expressed mRNAs with statistical significance were identified through Volcano Plot filtering.

The threshold required for the results to be considered significant was as follows: Q-value $\leq 0.01$ and absolute value of $\mid \log _{2}$ (Fold Change) $\mid \geq 1.0$.

\section{Functional enrichment analysis of DEGs}

Functional enrichment analysis was performed by functional annotation package "clusterProfiler" in $\mathrm{R}$ studio software (RStudio, Boston, MA, USA). GO and KEGG enrichment analysis was also conducted. For each enriched function term, $P$-value of enriched functions and the $P$-value by multiple testing corrections were calculated by "clusterProfiler" package in $\mathrm{R}$ studio software.

\section{Real-time quantitative PCR (qPCR)}

The extracted total RNA from the DRGs was reversetranscribed into cDNA using random hexamers primers (TaKaRa Bio Inc., Shiga, Japan) according to the manufacturer's instruction. The sequences of all primers used 
were shown in Table 1. qPCR was performed using DRG tissues from rats other than the ones used for RNA-Seq. Gapdh was used as an internal reference gene. qPCR was performed using the Fast Start Universal SYBR Green Master kit (TaKaRa Bio Inc.) with $25 \mu \mathrm{L}$ reaction system according to the manufacturer's protocol by CFX96 RealTime System (Bio-Rad Laboratories Inc., Hercules, CA, USA). Each reaction was performed in triplicates and normalized to Gapdh gene expression. The CT value of each well was determined using the CFX96 Real-Time System software and the average of the triplicates was calculated. The relative quantification was determined by the $\Delta \Delta \mathrm{CT}$ method. ${ }^{8,38}$

\section{Source of microarray data}

Pain genes (PG): The pain gene list was retrieved from a previous study using the same list to predict potential genes involved in mediating masseter inflammation-induced pain. ${ }^{13}$ This pain gene list contains 685 genes, which mainly includes human genes related to transmission of pain signals, central and peripheral inflammatory responses and pain mood and affect (see Table S2 for full list of genes).

Sensory neuronal genes (SN): The sensory neuronal gene list was retrieved from a previous study using magnetic cell sorting (MACS) to separate neuronal cells from nonneuronal cells from mouse DRG. ${ }^{48}$ In this study, Thakur et al demonstrated that MACS successfully segregated neuronal cells from nonneuronal cells from mouse DRGs. This procedure can enrich neuronal population up to $95 \%$ compared with $10 \%$ before sorting.

Transcriptome analysis further confirmed that many genes were enriched in the neuronal population collected through MACS. We selected genes that showed increase in expression by $>40 \%$ according to criterion described by Chung et $\mathrm{al}^{13}$ (see Table S2 for full list of genes).

TRPV1-lineage neuronal genes (VL): TRPV1-lineage neuronal genes list was derived from a recent study which identified a group of genes enriched in TRPV1 expressing nociceptive sensory neurons. ${ }^{24}$ By using mouse lines selectively labeling or ablating TRPV1 lineage neurons, Goswami et al identified a group of genes enriched in VL sensory neurons. We screened genes that are enriched $>3$-fold in VL neurons compared to non-VL neurons according to a study by Chung et $\mathrm{al}^{13}$ (see Table S2 for full list of genes).

SNL and SNI neuropathic microarray: Data was downloaded from GEO (Gene Expression Omnbius) dataset, at the website of https://www.ncbi.nlm.nih.gov/geo/. Two separate microarray datasets GSE30691 and GSE24431 were selected for the research. ${ }^{10,17}$ The GSE30691 datasets contained 56 DRG tissues from rats which evaluated gene changes in SNI, SNL and CCI neuropathic pain condition at different time points. We chose DRG samples collected

Table I Sequences of the primers used for qPCR validation of RNA-Seq data

\begin{tabular}{|c|c|c|c|}
\hline Gene name & Gene ID & Primer sequence $\left(5^{\prime}\right.$ to $\left.3^{\prime}\right)$ & Amplicon size (bp) \\
\hline Gapdh & 24,383 & $\begin{array}{l}\text { F: 5'-ACATGCCGCCTGGAGAAAC-3' } \\
\text { R: 5'-AGCCCAGGATGCCCTTTAGT-3' }\end{array}$ & 92 \\
\hline Hpd & 29,531 & $\begin{array}{l}\text { F:5'-GTTCAAAGCTTTCGAAGAGGAG-3' } \\
\text { R:5'-GCATCTTTACTCGAAACGGATC-3' }\end{array}$ & 229 \\
\hline Vip & 24,875 & $\begin{array}{l}\text { F:5'-CTTCTCCCAAGCAGAACTTCAG-3' } \\
\text { R:5'-TCATCCAACCTCACTGAAGAAG-3' }\end{array}$ & 207 \\
\hline Zbtb 16 & 353,227 & $\begin{array}{l}\text { F:5'-CACCAGCAAGATGTTTGAGATC-3' } \\
\text { R:5'-CTGTGTCATTGTCATCAGATGC-3' }\end{array}$ & 239 \\
\hline Ostn & 360,730 & $\begin{array}{l}\text { F:5'-CCTAGTGTTTAAAGGTCTGATGATG-3' } \\
\text { R:5'-TTACTGTAGCCTTCTGTCACAG-3' }\end{array}$ & 178 \\
\hline Sprrla & 499,660 & $\begin{array}{l}\text { F:5'-AGCAGAAGACAAAGCAGAAGTA-3' } \\
\text { R:5'-GGACTCATAAGCAGGATGGAAA-3' }\end{array}$ & 95 \\
\hline $11-24$ & 170,819 & $\begin{array}{l}\text { F:5'-TCACTCTTTAGTCCAAACGACA-3' } \\
\text { R:5'-GGAACGTCCAGTCTCACAAATA-3' }\end{array}$ & 149 \\
\hline II-6 & 25,205 & $\begin{array}{l}\text { F:5'-TGCACTGTCAGAAAACAATCTG-3' } \\
\text { R:5'-CCAGAGCAGATTTTCAATAGGC-3' }\end{array}$ & 105 \\
\hline Nps & $100,360,07 \mid$ & $\begin{array}{l}\text { F:5'-TGGTACACGTGATTTGGAAGTTA-3' } \\
\text { R:5'-CATTGCTTTGCTCTTCGAAATG-3' }\end{array}$ & 220 \\
\hline Cxcll3 & 498,335 & $\begin{array}{l}\text { F:5'-CGACTTTGGAAAGGTTGCTTGTA-3' } \\
\text { R:5'-ACACTGGATGAATAGGAAACGT-3' }\end{array}$ & 219 \\
\hline
\end{tabular}


from day 7 after SNI or SNL model establishment, which is the same as the time points of our CPIP model experiment, for comparison. Six DRG samples derived from three SNI and three corresponding sham rats and six DRG samples derived from three SNL and three corresponding sham rats post-day 7 were used for the subsequent analysis. Five samples of CFA model rats and five naïve samples as control were included in GSE24551 dataset and analyzed. Differentially expressed genes from these microarray datasets were screened based on criteria of Q-value $\leq 0.01$ and absolute value of $\mid \log _{2}$ (Fold Change) $\mid \geq 1.0$.

\section{Protein-protein interaction (PPI) network analysis}

The search tool for the retrieval of interacting genes (STRING) is used to provide information regarding predicted and experimental interactions of proteins and the prediction method of this database is from neighborhood, gene fusion, co-occurrence, co-expression experiments, databases, and text mining. By setting the Combination score $>0.4$ as the reliability threshold value, the web based STRING database (http://string-db.org/) was used to produce PPI predictions after uploading the union gene list to the search bar. ${ }^{23}$ Based on the interplayed relationships, a PPI network was established and then visualized using the Cytoscape software. ${ }^{44}$ The connectivity degree of each protein, namely the number of proteins it connected, was calculated to evaluate its importance in this network.

\section{Statistical analysis}

qPCR and behavioral results were analyzed by unpaired Student's $t$-test or two-way ANOVA followed by Tukey's post hoc test. Data in graphs are expressed as means \pm SEM. Comparison is considered significantly different if the $P$-value is less than 0.05 .

\section{Results}

\section{The establishment of the CPIP rat model}

The rat CPIP model is a frequently used animal model in laboratory to mimic human CRPS-I. We followed the methods described by Coderre et al to establish the rat CPIP model. An O-ring tourniquet was applied to the right ankle joint for 3 hours to reduce the blood flow to the hind paw. ${ }^{16}$ During the treatment, the hind paw showed cyanosis, indicating tissue hypoxia (Figure 1A, left panel). Ten minutes after the reperfusion, the ipsilateral hind paw showed obvious edema compared with contralateral hind paw (Figure 1A, middle panel). Seven days later, the treated hind paw showed signs of excoriation and dryness (Figure 1A, right panel). The ipsilateral hind paw showed obvious edema after the treatment but gradually returned to normal 72 hours later (Figure 1B). We measured the pain responses of CPIP model rats for a consecutive 14 days. As shown in Figure $1 \mathrm{C}$ and D, CPIP model rats exhibited rapid and persistent thermal and mechanical hyperalgesia compared with Sham group rats. Thermal and mechanical hyperalgesia both appeared 1 day after model establishment and lasted until 14 days of our observation time frame. We sacrificed the rats on day 7 , a time point when rats exhibited stable pain responses and then harvested ipsilateral L4-6 dorsal root ganglia (DRGs) for RNA-Seq.

\section{Overview of gene expression profiles of DRGs after CPIP by RNA-Seq}

We then used RNA-Seq to explore gene expression profiles of ipsilateral L4-6 DRGs 7 days after CPIP. The sequencing produced approximate 24.1 million raw reads per sample and the clean reads ratio was nearly $99.0 \%$ (Table 2). More than $89 \%$ of bases had a quality score $\geq$ Q30 and over $95 \%$ of the clean reads data were mapped to the rat genome (Table 2). Eventually, 15,250 genes were successfully mapped and identified from RNA-Seq. Next, we utilized the hierarchical clustering analyses to get an overview of gene expression profiles of CPIP model rats compared with Sham rats. Hierarchical clustering analysis of all genes showed that three3 CPIP model samples and three3 Sham group samples were clustered in separate groups (Figure 2A). The clear segregation and clustering of the sequencing data indicated a distinct RNA expression profile existed between CPIP and Sham groups but not within groups. We then filtered out the DEGs with criteria of fold change $\geq 2$ and q-value $\leq 0.01$. The volcano plot indicated the number of DEGs in the CPIP group compared with the Sham group (Figure 2B). A total of 295 DEGs (195 up- and 100 downregulated) were identified (Figure 2B). The identified DEGs were further summarized and illustrated in a heat map with cluster analysis, which indicated a high level of consistency in either CPIP or Sham group samples (Figure 2C). The above data demonstrated that the rat CPIP model was accompanied with significant gene changes in ipsilateral DRGs. 
A Ischemia
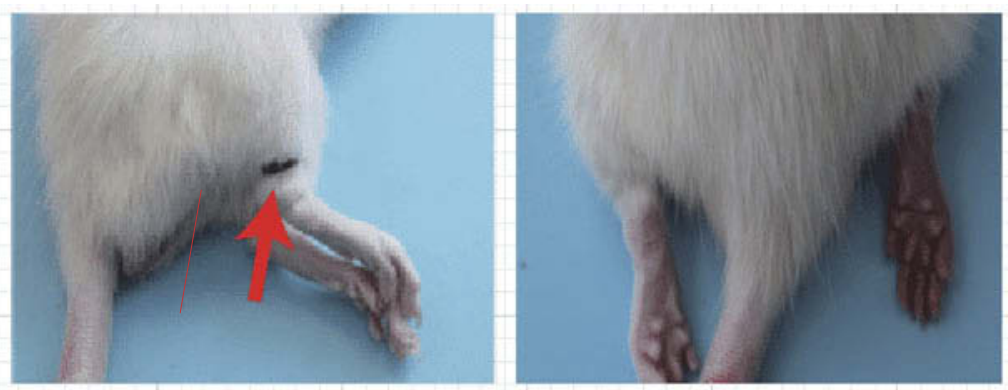

B

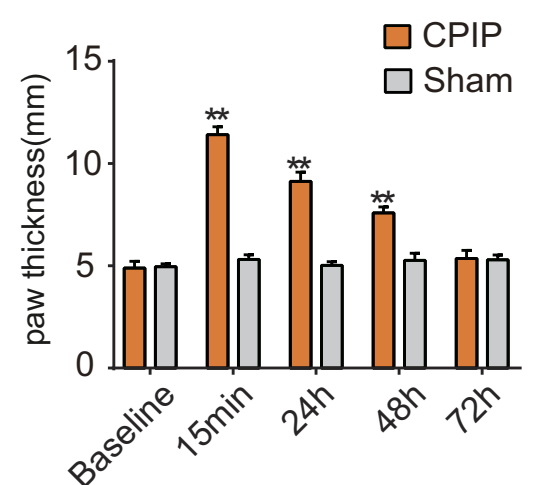

C

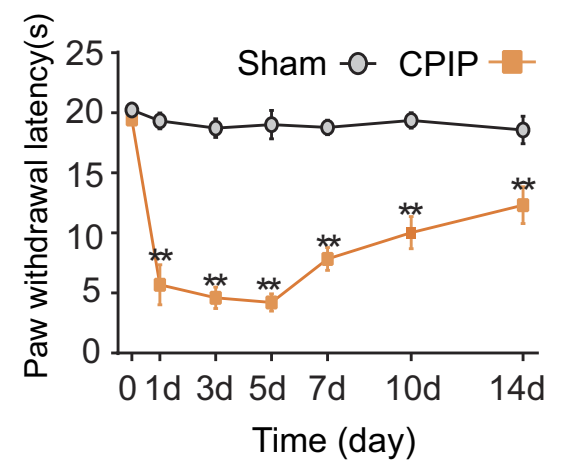

Post 7 day

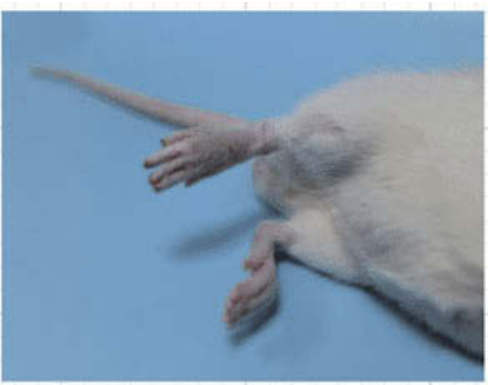

D

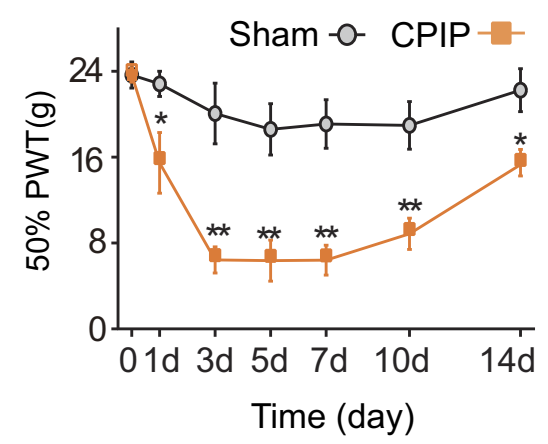

Figure I The CPIP rat model exhibits persistent mechanical and thermal hyperalgesia. (A) Representative photographs of rat hind paw taken during CPIP model establishment, 10 minutes and 7 days after model establishment. The red arrow indicates the $O$ ring clamped right above the ankle. (B) Thickness evaluation of ipsilateral hind paws of CPIP and Sham group rats. (C) Paw withdraw latency of ipsilateral hind paws of Sham and CPIP group rats. (D) $50 \%$ paw withdraw threshold of ipsilateral hind paw of Sham and CPIP group rats. $N=6$ rats/group. $* P<0.05$ and $* * P<0.01$ vs Sham group.

Abbreviation: CPIP, chronic post-ischemic pasin.

Table 2 The information of total reads and mapping ratio for Sham and CPIP groups in RNA-Seq

\begin{tabular}{|l|l|l|l|l|l|}
\hline Sample & $\begin{array}{l}\text { Total raw reads } \\
\text { (Mb) }\end{array}$ & $\begin{array}{l}\text { Total clean reads } \\
\text { (Mb) }\end{array}$ & $\begin{array}{l}\text { Clean reads Q30 } \\
\text { (\%) }\end{array}$ & $\begin{array}{l}\text { Clean reads ratio } \\
\text { (\%) }\end{array}$ & $\begin{array}{l}\text { Total mapping ratio } \\
\text { (\%) }\end{array}$ \\
\hline Sham I & 24.07 & 23.95 & 89.93 & 99.50 & 95.25 \\
Sham 2 & 24.08 & 23.96 & 89.65 & 99.51 & 95.15 \\
Sham 3 & 24.07 & 23.95 & 89.87 & 99.48 & 95.21 \\
CPIP I & 24.08 & 23.99 & 89.65 & 99.64 & 95.10 \\
CPIP 2 & 24.08 & 23.97 & 89.89 & 99.54 & 95.16 \\
CPIP 3 & 24.08 & 23.82 & 89.93 & 98.93 & 95.18 \\
\hline
\end{tabular}

\section{Analysis of DEGs in DRGs of CPIP model}

Among the DEGS we have obtained, some of the genes are well established to be involved in inflammation and pain processes. These genes include: Vip (vasoactive intestinal polypeptide, fold change $=261.4$ ), IL6 (interleukin- 6 precursor, fold change $=163.1)$, Cxcl13 $(\mathrm{C}-\mathrm{X}-\mathrm{C}$ motif chemokine 13 precursor, fold change $=5.6$ ), $\mathrm{Ccl} 2$ (C-C motif chemokine 2 precursor, fold change $=2.7$ ), Nlrp3 (NLR family pyrin domain containing 3, fold change $=2.6$ ) and $M M P 9$ (matrix metalloproteinase-9 precursor, fold change $=2.4$ ), etc. In addition, many other genes, whose roles in inflammation and pain have not yet been identified, were dramatically altered as well. We analyzed the data and found that 38 genes showed expression changes more than 10-fold, with 37 upregulated genes and one downregulated gene, such as Sprrla (small proline-rich protein 1A, fold change=477.4), 
A

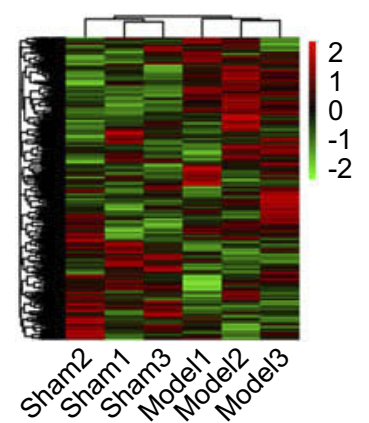

B

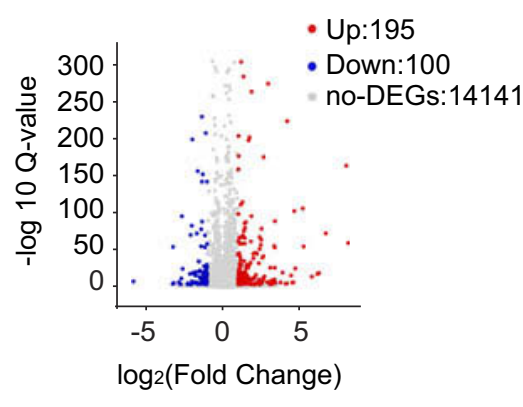

C

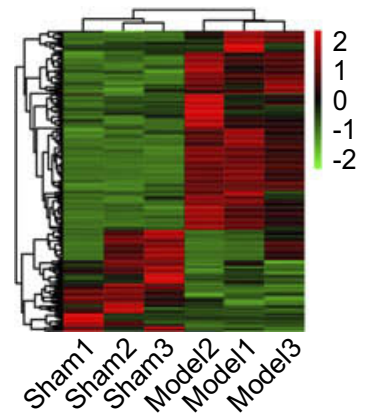

Figure 2 RNA-Seq reveals transcriptome profile of gene expression changes in DRGs induced by CPIP. (A) Heat map illustration of hierarchical clustering of overall gene expression profile of DRGs from Sham and CPIP group rats. (B) Volcano plot showing gene expression profiles in DRGs of CPIP group compared with Sham group. Red and blue spots indicate up- and downregulated DEGs, respectively, whereas grey spots indicate non-DEGs. (C) Heat map illustration of hierarchical clustering of DEGs from CPIP and Sham groups.

Abbreviations: RNA-Seq, RNA-sequencing; DRGs, dorsal root ganglions; CPIP, chronic post-ischemic pain; DEGs, differentially expressed genes.

Crisp1 (cysteine-rich secretory protein 1 precursor, fold change $=451.2$ ), Lcelf (late cornified envelope 1F, fold change $=372.4$ ); 39 genes showed expression changes between 5- and 10-fold, which include 29 upregulated and 10 downregulated genes. Tables 3 and 4 illustrated the detailed information of the top 20 up- and 20 downregulated DEGs.

\section{Validation of RNA-Seq data by real-time quantitative PCR (qPCR)}

We proceeded to verify the RNA-Seq data by qPCR. Two upregulated genes ( $H p d$ and $V i p$ ) and two downregulated genes (Zbtb16 and Ostn) from DEGS were randomly selected and analyzed by qPCR. qPCR results indicated that the expression of $H p d$ and Vip were significantly

Table 3 The detailed information of the top 20 upregulated DEGs

\begin{tabular}{|c|c|c|c|c|c|}
\hline $\begin{array}{l}\text { Upregulated } \\
\text { Gene }\end{array}$ & Gene ID & Location & $Q$ value & $\begin{array}{l}\log _{2} \text { fold-change } \\
\text { (CPIP/Sham) }\end{array}$ & Official gene name (NCBI) \\
\hline Sprrla & 499,660 & $2: 192,669,143-192,671,059$ & I. I7E-45 & 8.89909 & Small proline-rich protein IA \\
\hline Crispl & 64,827 & $9: 24,356,577-24,383,679$ & I.27E-43 & 8.817702 & Cysteine-rich secretory protein I \\
\hline Lcelf & 499,663 & $2: 192,990,607-192,992,193$ & $2.56 \mathrm{E}-37$ & 8.54087 & Late cornified envelope IF \\
\hline Hamp & 84,604 & $\mathrm{I}: 89,368,02 \mathrm{I}-89,369,960$ & $5.19 \mathrm{E}-59$ & 8.155005 & Hepcidin antimicrobial peptide \\
\hline Vip & 117,064 & $\mathrm{I}: 42,169,307-42,177,582$ & $2.17 \mathrm{E}-163$ & 8.034563 & Vasoactive intestinal peptide \\
\hline 116 & 24,498 & $4: 3,043,231-3,047,807$ & $8.8 \mathrm{IE}-19$ & 7.350197 & Interleukin 6 \\
\hline Serpinb2 & 60,325 & $|3: 27,449,907-27,463,0| 5$ & $4.85 \mathrm{E}-72$ & 6.701509 & Serpin family B member 2 \\
\hline Npy & 24,604 & $4: 79,557,856-79,565,059$ & 0 & 6.391931 & Neuropeptide $Y$ \\
\hline $\operatorname{Tgm} I$ & 60,335 & $|5: 34,378| 36-34,393,150$, & 0 & 6.325616 & Transglutaminase I \\
\hline Hpd & 29,531 & $12: 38,828,606-38,839,956$ & $1.17 \mathrm{E}-18$ & 6.258274 & 4-hydroxyphenylpyruvate dioxygenase \\
\hline $5 / c 30 a 3$ & 366,568 & $6: 26,629,752-26,650,106$ & $1.54 \mathrm{E}-17$ & 6.160094 & Solute carrier family 30 member 3 \\
\hline 1124 & 170,819 & $|3: 47,6| 8,45 \mid-47,623,849$ & $7.18 \mathrm{E}-14$ & 5.791706 & Interleukin 24 \\
\hline RTI-NI & 24,748 & Unknown & $2.33 \mathrm{E}-07$ & 5.765234 & RTI class Ib, locus NI \\
\hline Serpina3n & 24,795 & $6: 128,073,344-28,080,878$ & 0 & 5.390968 & $\begin{array}{l}\text { Serine (or cysteine) peptidase inhibitor, } \\
\text { clade } A \text {, member } 3 \mathrm{~N}\end{array}$ \\
\hline Stac2 & 363,674 & $10: 85,988,367-86,004,209$ & 0 & 5.345099 & $\mathrm{SH} 3$ and cysteine rich domain 2 \\
\hline Ucn & 29,151 & $6: 26,602,144-26,602,974$ & $2.94 \mathrm{E}-54$ & 5.264588 & Urocortin \\
\hline Cldn 4 & 304,407 & $\mid 2: 24,761,210-24,763,008$ & $1.49 \mathrm{E}-105$ & 5.205793 & Claudin-4 \\
\hline Nps & $100,360,07 \mid$ & $|: 207,508,4| 4-207,5 \mid 2,195$ & $2.98 \mathrm{E}-04$ & 4.817702 & Neuropeptide S \\
\hline Kbtbd6 & 306,073 & $|5-6|, 73|, 574-6|, 733,33 \mid$ & $3.13 \mathrm{E}-04$ & 4.808397 & $\begin{array}{l}\text { Kelch repeat and BTB domain- } \\
\text { containing protein } 6\end{array}$ \\
\hline Vten $I$ & 295,322 & $2: 203,200,435-203,274,264$ & $1.16 \mathrm{E}-24$ & 4.724592 & $\begin{array}{l}\text { V-set domain-containing T-cell activa- } \\
\text { tion inhibitor I }\end{array}$ \\
\hline
\end{tabular}


Table 4 The detailed information of the top 20 downregulated DEGs

\begin{tabular}{|c|c|c|c|c|c|}
\hline $\begin{array}{l}\text { Downregulated } \\
\text { Gene }\end{array}$ & Gene ID & Location & Q-value & $\begin{array}{l}\log _{2} \text { fold change } \\
\text { (CPIP/sham) }\end{array}$ & Official gene name (NCBI) \\
\hline LOC367,975 & 367975 & $X: 124,156,649-124,162,089$ & I.65E-07 & -5.78874 & Similar to B-cell translocation gene I \\
\hline Ostn & 360,730 & ||$: 76,85 \mid, 923-76,888,140$ & $2.04 \mathrm{E}-04$ & -3.23758 & Osteocrin \\
\hline Msln & 60,333 & $10: 15,119,700-15,129,129$ & I.3IE-53 & -3.21847 & Mesothelin \\
\hline |gl|I & $100,360,919$ & | I:88,388,378-88,39|,524 & $1.04 \mathrm{E}-05$ & -3.15958 & $\begin{array}{l}\text { Immunoglobulin lambda-like poly- } \\
\text { peptide I }\end{array}$ \\
\hline Csapl & $|7|,|6|$ & $10: 13,230,181-13,230,577$ & $1.25 \mathrm{E}-06$ & -2.83765 & Common salivary protein I \\
\hline Atp Ib4 & 84,396 & $X: 124,63 \mid, 544-124,652,520$ & $2.4 \mathrm{IE}-05$ & $-2.7970 \mathrm{I}$ & $\begin{array}{l}\text { ATPase } \mathrm{Na}+/ \mathrm{K}+\text { transporting family } \\
\text { member beta } 4\end{array}$ \\
\hline Adamts 19 & 361,332 & $|8: 53,9| 5,807-54,099,856$ & 4.67E-04 & -2.72662 & $\begin{array}{l}\text { ADAM metallopeptidase with } \\
\text { thrombospondin type I motif, } 19\end{array}$ \\
\hline Ucp3 & 25,708 & I:165,482,9|2-165,495,895 & $2.14 \mathrm{E}-1 \mid$ & -2.67997 & Uncoupling protein 3 \\
\hline LOC688,090 & 688090 & Unknown & $6.58 \mathrm{E}-95$ & -2.6443 & $\begin{array}{l}\text { Similar to RTI class II histocompat- } \\
\text { ibility antigen, B-I beta chain } \\
\text { precursor }\end{array}$ \\
\hline$T n n$ & 304,913 & $|3: 77,829,384-77,896,83|$ & $2.34 \mathrm{E}-24$ & -2.60109 & Tenascin N \\
\hline Myh4 & 360,543 & $10: 53,778,456-53,801,605$ & 0 & -2.43883 & Myosin heavy chain 4 \\
\hline Cxclll & 305,236 & $14: 17,195,395-17,198,170$ & 3.57E-05 & -2.26976 & C-X-C motif chemokine ligand II \\
\hline Ly6al & 362,935 & $7: 116,557,519-116,561,058$ & $8.20 \mathrm{E}-04$ & -2.2694 & $\begin{array}{l}\text { Lymphocyte antigen } 6 \text { complex, } \\
\text { locus A-like }\end{array}$ \\
\hline$R T I-D b 2$ & 24,981 & $20: 4,106,136-4,126,103$ & $2.85 \mathrm{E}-17$ & -2.12149 & RTI class II, locus Db2 \\
\hline Myh2 & 691,644 & $|0: 53,7| 1,895-53,738,164$ & $1.17 \mathrm{E}-69$ & -2.07528 & Myosin heavy chain 2 \\
\hline$V g \| 12$ & 309,772 & $20: 33,077,106-33,082,962$ & $9.62 \mathrm{E}-04$ & -2.04855 & Vestigial-like family member 2 \\
\hline Artl & 308,873 & I:167,202,064-167,206,615 & $1.20 \mathrm{E}-07$ & -1.99595 & ADP-ribosyltransferase I \\
\hline Zbtb 16 & 353,227 & $8: 52,980,226-53,146,765$ & 4. $13 \mathrm{E}-82$ & -1.99516 & $\begin{array}{l}\text { Zinc finger and BTB domain con- } \\
\text { taining } 16\end{array}$ \\
\hline$L O C I 00,134,87 I$ & $100|3487|$ & I:168,957,460-168,958,836 & $8.31 \mathrm{IE}-198$ & -1.96213 & Beta globin minor gene \\
\hline Ifitl & 56,824 & $\mathrm{I}: 252,944,105-252,946,170$ & $7.38 \mathrm{E}-19$ & -1.93491 & $\begin{array}{l}\text { Interferon-induced protein with tet- } \\
\text { ratricopeptide repeats I }\end{array}$ \\
\hline
\end{tabular}

increased, whereas Zbtb16 and Ostn were significantly decreased in DRGs of the CPIP model group compared with the Sham group (Figure 3A and B). These qPCR results were consistent with RNA-Seq data. We further evaluated some representative genes whose roles in inflammation or pain had been documented, such as $I l 24$, Il6, Nps and Cxcll3 by qPCR. As a control, we also included Sprrla, the gene which showed the highest
A

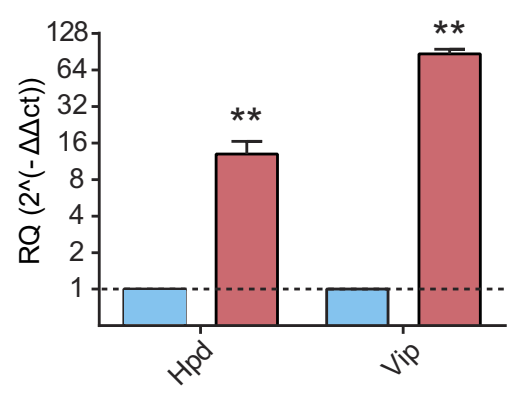

B

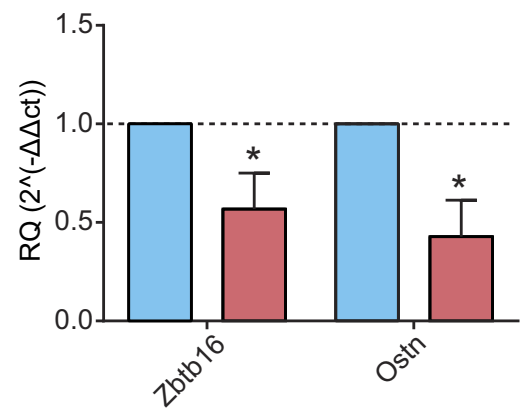

C

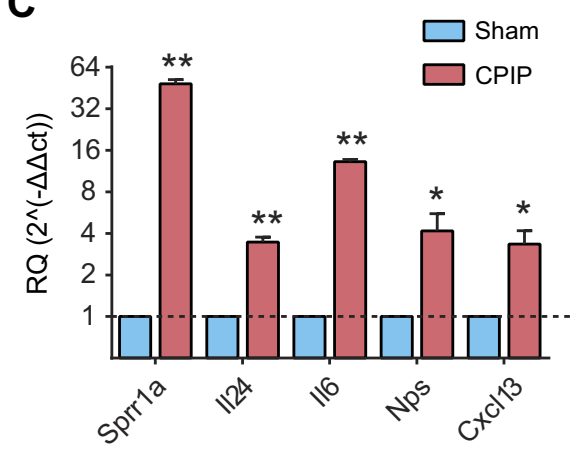

Figure 3 The validation of RNA-Seq results using qPCR. (A) The expression of two randomly selected upregulated DEGs from RNA-seq detected by qPCR. (B) The expression of two randomly selected downregulated DEGs from RNA-seq detected by qPCR. (C) The expression of representative genes in inflammation and pain detected by $q P C R$. $N=6$ rats/group. $* P<0.05, * * P<0.01$ vs Sham group.

Abbreviations: RNA-Seq, RNA-sequencing; DEGs, differentially espressed genes. 
increase fold in RNA-Seq, in our test. qPCR results showed that the expression of Il24, Il6, Nps, Cxcll3 as well as Sprrla were all significantly increased in DRGs of the CPIP model group compared with the Sham group (Figure 3C), which was consistent with RNA-Seq data Therefore, the qPCR assay confirmed the reliability of the RNA-Seq data.

\section{Pathway analysis of DEGs of CPIP model}

To further investigate the molecular mechanisms underlying CRPS-I, we carried out GO analysis of the DEGs in DRGs of the CPIP model and the Sham groups. Results obtained from the GO analysis indicated that the most significantly enriched biological process of upregulated DEGs in the CPIP model group was response to lipopolysaccharide (LPS), inflammatory response, defense response to fungus, neuropeptide signaling pathway and positive regulation of ERK1 and ERK2 cascade, etc (Figure 4A). The most significantly enriched molecular function of upregulated DEGs in the CPIP model group was G-protein coupled receptor binding, cytokine activity, and calcium ion binding, etc (Figure 4B). The most significantly enriched cellular function of upregulated DEGs in the CPIP model group was extracellular space and extracellular region, etc (Figure 4C). In addition, the most

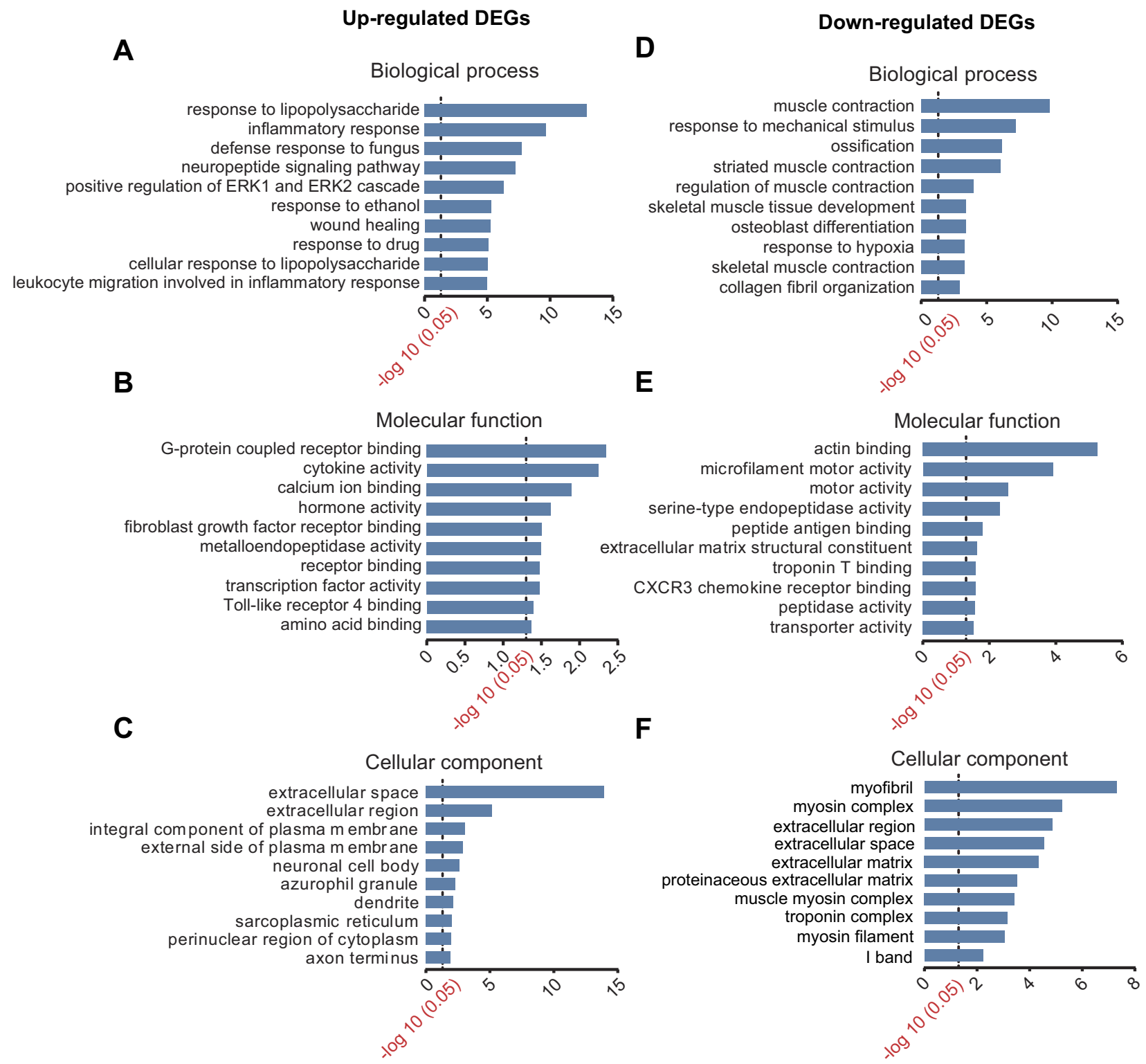

Figure 4 GO pathway analysis of DEGs. (A-C) The top 10 significant biological processes, molecular functions and cellular components of upregulated DEGs. (D-F) The top 10 significant biological processes, molecular functions and cellular components of downregulated DEGs. The dotted line indicated $P$-value of 0.05 .

Abbreviations: GO, gene ontology; DEGs, differentially expressed genes. 
significantly enriched biological process of downregulated DEGs in CPIP model group was muscle contraction, response to mechanical stimulus, ossification, striated muscle contraction, etc (Figure 4D). The most significantly enriched molecular function of downregulated DEGs in the CPIP model group was actin binding, microfilament motor activity, and motor activity, etc (Figure 4E). The most significantly enriched cellular function of downregulated DEGs in the
CPIP model group was myofibril, myosin complex, and extracellular region, etc (Figure 4F).

We further performed gene enrichment analysis using KEGG pathway analysis. As shown in Figure 5A, the upregulated DEGs in DRGs of the CPIP model were mainly involved in phagosome, cell adhesion molecules, herpes simplex infection, rheumatoid arthritis, and TNF signaling pathway, etc. The downregulated DEGs in DRGs

\section{A}

Up-regulated DEGs

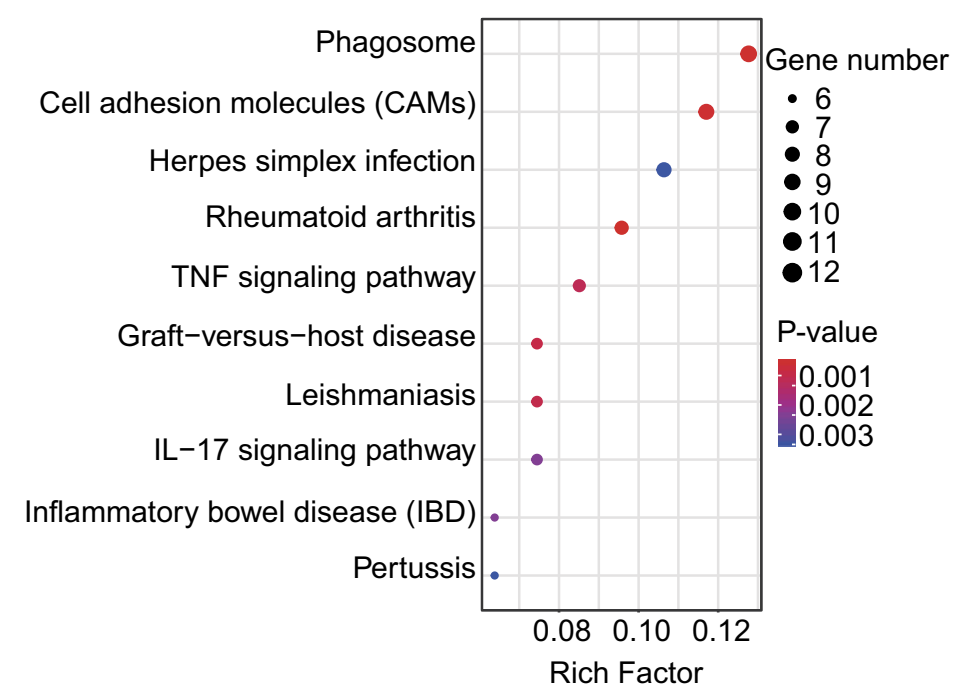

B

\section{Down-regulated DEGs}

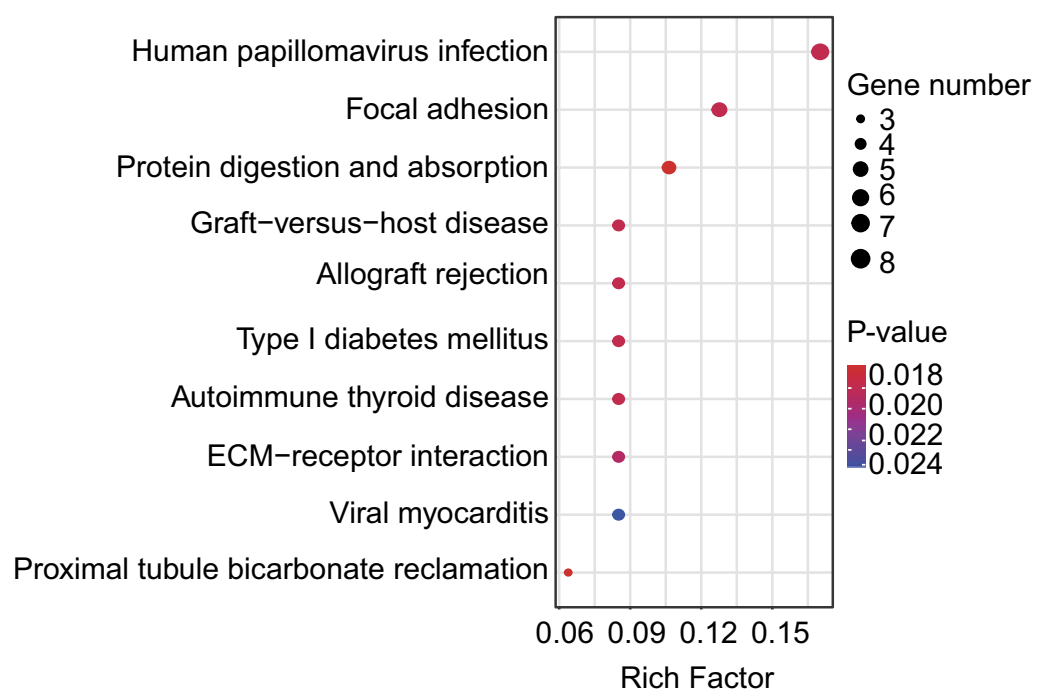

Figure 5 KEGG pathway analysis of DEGs. (A) Bubble plots showing the top 10 significant pathways for upregulated DEGs. (B) Bubble plots showing the top I0 significant pathways for downregulated DEGs. Larger bubbles indicate higher number of genes. The color of each bubble reflects significance ( $P$-value).

Abbreviations: KEGG, Kyoto Encyclopedia of Genes and Genomes; DEGs, differentially expressed genes. 
of the CPIP model mainly involved human papillomavirus infection, focal adhesion, and protein digestion and absorption, etc (Figure 5B).

To focus our analysis on the genes more related with pain and nociception, we searched previously published microarray datasets about genes involved in pain processing (PG), genes enriched in small-diameter primary sensory DRG neurons (SN) and genes enriched in TRPV1-lineage nociceptors (VL) from previously published datasets. ${ }^{11,19,37}$ We found that 37 DEGs are related to pain processing and Vip, Il6 and Illa are among the pain-related genes that are most highly upregulated (Figure 6A, Tables S2 and S3). Twelve DEGs are related to small-diameter primary sensory DRG neurons and Sprrla showed the highest upregulation (Figure 6A, Tables S2 and S3). Six genes are related to TRPV1-lineage nociceptor and Gal showed the highest upregulation (Figure 6A, Tables S2 and S3). We mapped our DEGs with these datasets and found that over 47 genes out of 295 DEGs $(15.9 \%$ in total) were related to pain processing and nociception (Figure 6A). We performed PPI analysis of these 47 genes and found that the major hub genes deduced from PPI analysis consisted of Il6, Ccl2, Jun, Mmp9, Fgf2, Cck, Itgam and Tlr2, etc (Figure 6B).

\section{Comparison of our RNA-Seq dataset with other published datasets of neuropathic or inflammatory pain models}

The CPIP rat model is an animal model that mimics human CRPS-I, a neuropathic pain condition. However, it is still not known whether the CPIP model shows any typical neuropathic pain-related gene expression profiles. Therefore, we compared our CPIP RNA-Seq data with the microarray data obtained from two well-known neuropathic pain models, namely, the rat models of SNI (spared nerve injury) and SNL (spinal nerve ligation). The same criteria (fold change $\geq 2, q \leq 0.01$ ) were imposed upon both SNI and SNL microarray datasets for identifying DEGs. We observed that the CPIP model group had an overlap of 34 and 41 genes with SNI and SNL models, respectively, which accounts for $11.5 \%$ and $13.9 \%$ of all DEGs of the CPIP model. In addition, the three groups had a core set of 19 genes that were overlapped (Figure 7A, Table S4). For the CPIP model, the core set of genes included 17 upregulated genes and two downregulated genes (Table S4). We further compared the DEGs of the CPIP model with the CFA model, a well-established inflammatory pain model. We found that seven genes in all were overlapped between these two groups, which accounts for only $2.4 \%$ of all DEGs of the CPIP model (Figure 7B). Besides, only thee out of the seven genes showed uniform changes in CPIP and CFA models (Table S5). These findings suggested that the CPIP model exhibited more changes in neuropathic-pain related genes than inflammatory-pain related genes.

\section{Discussion}

In the present study, we performed transcriptome profiling of the DRGs which innervate the affected hind limb of the CPIP model rats by means of RNA-Seq. We analyzed the genes that are differentially regulated and confirmed their expression by qPCR. We further sorted out the genes that are possibly involved in pain mechanisms or enriched in nociceptors. Lastly, by comparing with published microarray datasets of rat neuropathic and inflammatory pain models, we identified key pathways shared by the rat CRPS-I model with two other neuropathic pain models. Distinct patterns of gene expression in different levels of the nociceptive system are critically involved in the development and maintenance of neuropathic pain. ${ }^{29,36}$ Therefore, we presented by far, the first study to profile the transcriptome changes and key pathways in the primary sensory ganglia of a rat model of CRPS-I using RNA-Seq.

In this study, a rat model of CPIP was used to mimic CRPS-I. Ischemia/reperfusion is among one of the possible factors leading to CRPS-I. ${ }^{4,15}$ It is reported that around $45 \%$ and $70 \%$ of CRPS-I patients develop signs of thermal and mechanical hypersensitivity, respectively. Thermal and mechanical hypersensitivities are among the major symptoms affecting CRPS-I patients. ${ }^{25,42,43,47}$ A recent study demonstrated CPIP model rats developed obvious signs of thermal hyperalgesia, which correlates with our present findings. ${ }^{46}$ Therefore, the CPIP rat model could be used as a preclinical animal model for studying mechanisms underlying both thermal and mechanical hypersensitivity of CRPS-I patients.

According to GO analyses of the DEGs in DRGs, we discovered that the significantly enriched biological processes and molecular functions of upregulated genes in CPIP vs Sham groups were mostly involved in response to LPS, inflammation, G-protein coupled receptor binding, cytokine activity, and neuropeptide signaling. These findings suggest that neuroinflammation is likely to be a predominant process involved in the pathophysiology of the CPIP model. This result also coincided with 
A

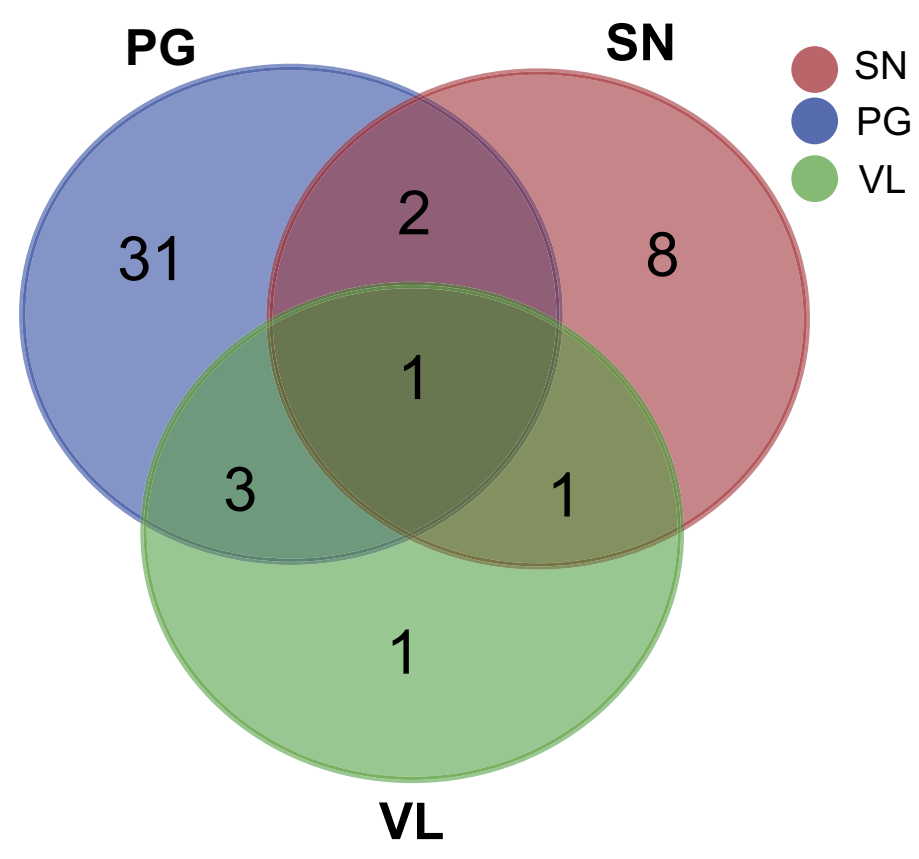

B

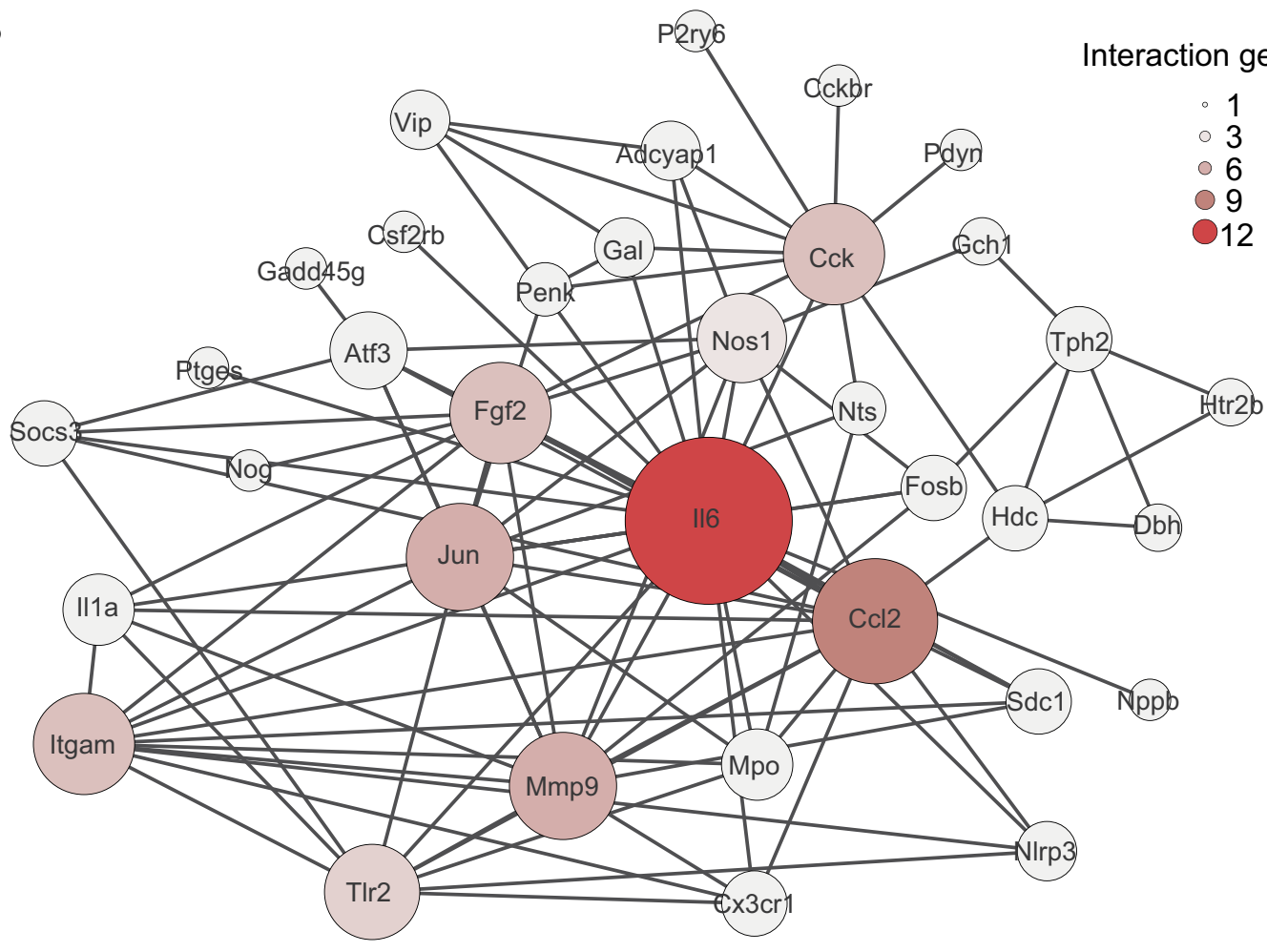

Figure 6 Overlapping of DEGs with genes involved in pain processing and nociception. (A) Venn diagram showing the overlapping of DEGs with genes involved in pain processing (PG, blue color), genes enriched in small-diameter DRG neurons (SN, pink color) and genes enriched in TRPVI-lineage nociceptors (VL, green color). (B) PPI network analysis of the DEGs overlapped with PG, SN and VL gene datasets. Larger circles and deeper colors reflect more interactions.

Abbreviations: DEGs, differentially expressed genes; DRG, dorsal root ganglion; PPI, protein-protein interaction. 
A

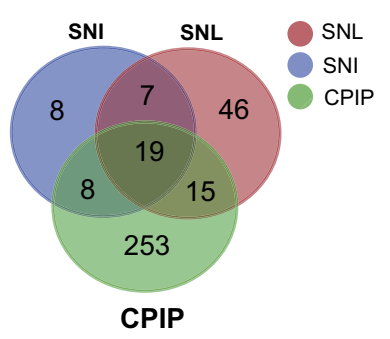

B

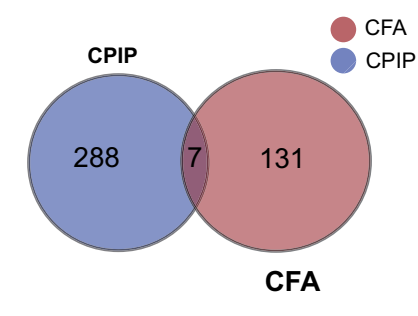

Figure 7 Comparison of our present CPIP RNA-Seq datasets with other published microarray datasets of neuropathic and inflammatory pain models. (A) Venn diagram showing the overlapping of DEGs in DRGs from CPIP rat model with rat model of SNI and SNL neuropathic pain. (B) Venn diagram showing the overlapping of DEGs in DRGs from CPIP rat model with rat model of CFA inflammatory pain. Abbreviations: CPIP, chonic post-ischemic pain; RNS-Seq, RNA-sequencing; DEGs, differentially expressed genes; DRGs, dorsal root ganlions.

previous studies showing that neuroinflammation, in terms of glial activation, immune cell infiltration, and inflammatory mediator release in the sensory system, is critically involved in neuropathic pain mechanisms. In addition, positive regulation of ERK1 and ERK2 cascade was also among the most significantly enriched pathways of upregulated genes by GO analysis. In fact, ERK1 and ERK2 cascade has been suggested to play a crucial role in mediating neuropathic pain as well as many other pain conditions. ${ }^{7,18,51}$ The above findings suggest that targeting neuroinflammation and ERK1/2 cascade may represent effective approaches to treat CRPS-I.

GO functional analysis also showed that downregulated DEGs might associate with muscle contraction, response to mechanical stimulus, ossification, striated muscle contraction, etc. At this time, we have no idea how these processes might be related with neuropathic pain or DRG function. But we also noticed that similar biological process, like skeletal muscle contraction, also showed up in the downregulated gene list of GO analysis in a study investigating DEGs in spinal cord dorsal horn upon SNL neuropathic pain. ${ }^{31}$ But how the above biological processes are related with CPIP-induced pain or DRG functions needs to be further investigated.

KEGG analysis shows that most of the upregulated genes are involved in phagosome. We noticed that phagosome was also observed in another study investigating DEGs in spinal dorsal horn involved in CCI-induced neuropathic pain model. ${ }^{21}$ One of the genes involved in phagosome is Itgam, which is a marker for macrophage. Neuropathic pain is usually accompanied with infiltration of macrophages in peripheral sensory ganglia. ${ }^{19,52}$ The infiltrated macrophages produce oxidative stress and release ROS products, including $\mathrm{H}_{2} \mathrm{O}_{2}$ and lipid peroxidation products, which in turn activate TRPA1 and cause pain. ${ }^{1,37}$ Therefore, our data suggest that macrophages may be involved in the pain mechanisms of CPIP model. In addition, KEGG analysis also shows that most of the downregulated genes are involved in human papillomavirus infection. However, at this moment we do not know how these genes are related to CPIP-induced pain or DRG functions and further studies are needed to verify this finding.

The pathophysiological mechanisms of CRPS still remain poorly understood. Studies have been undertaken to monitor the inflammatory mediators in local blister fluid of CRPS patients. ${ }^{6}$ It is found that levels of IL- 6 and TNF- $\alpha$ are highly elevated in blister fluid of CRPS patients. ${ }^{6}$ In our study, we found that IL-6 is among one of the most upregulated pain-related genes in DRGs of the CPIP model rats. Our subsequent qPCR results confirmed a more than 10-fold upregulation of IL6 in DRGs of the CPIP model rats. Furthermore, by performing PPI network analysis, we found that IL6 was the hub gene that showed the most interactions with other pain or nociception-related genes. Recently, the expression levels of IL-6 and its receptor have been found to be upregulated in DRGs and spinal cord in pathological pain and nerve injury conditions. ${ }^{26,34,45,54}$ Moreover, application of IL-6 neutralizing antibody ameliorated these pain-related behaviors. ${ }^{26,34,41}$ Therefore, our findings, together with others, suggest that the upregulation of IL-6 in both primary sensory ganglia and local tissue may play an important role in mediating CRPS-I.

A genome-wide expression profiling in the blood from CRPS patients using microarray has been performed and identified MMP9 to be significantly upregulated in the blood samples of CRPS-I patients. ${ }^{32}$ In our study, MMP9 is also among the DEGs we identified that are significantly upregulated in the DRGs. MMP-9 belongs to a family of extracellular proteases named matrix metalloproteinases that play crucial roles in neuroinflammation and neuropathic pain in the nerve system. ${ }^{20}$ MMP9 expression is dramatically increased in DRGs of rats undergone sciatic nerve crush and MMP-9 gene deletion can protect nerve fibers from demyelination and reduce neuropathic pain after injury. ${ }^{11}$ MMP9 genedeficient mice showed reduced neuropathic pain response. ${ }^{33}$ Therefore, our study suggested that upregulation of MMP9 gene may underlie the pain mechanisms of CRPS-I.

We also observed obvious upregulation of CXCL13 gene in the DRGs of CPIP model rats by RNA-Seq. CXCL13 is a B lymphocyte chemoattractant and activates CXCR5 receptor in the immune system. ${ }^{53}$ CXCL13 was recently found to be the most upregulated gene among all chemokines in the spinal cord tissue after SNL-induced neuropathic 
pain. ${ }^{30}$ Genetic knockout Cxcr5 can alleviate neuropathic pain and reduce spinal glial activation. ${ }^{30}$ More recently, CXCL13/CXCR5 signaling was found to be upregulated in DRG neurons and participate in inflammatory pain via enhancing Nav1.8 current density in DRG neurons through acting on p38 MAP kinase. ${ }^{50}$ These findings demonstrated a pivotal role of CXCL13/CXCR5 in mediating neuropathic and inflammatory pain. ${ }^{53}$ Our qPCR result further confirmed that Cxcl13 mRNA expression was significantly increased in DRGs of CPIP model rats. Therefore, our study suggests that CXCL13 may be another promising pharmacological target for CRPS-I treatment.

CRPS-I patients usually developed obvious heat and cold pain hypersensitivity and increased responses to capsaicin stimulation. ${ }^{25,47}$ TRPV1-expressing DRG neurons are mainly nociceptive sensory neurons and play crucial roles in transuding signals arising from exposure to painful heat, noxious cold, inflammatory and chemical stimuli. $^{14,24,40}$ Therefore, it is likely that TRPV1-lineage DRG neurons may play a role in mediating CRPS-I pain symptom. Therefore, we mapped our identified DEGs with the recently published datasets of TRPV1-lineage genes, as well as pain-related genes, and genes enriched in sensory neurons. ${ }^{13,24,48}$ This approach can allow us to better deduce the possible contributions of these genes to the pain mechanisms of CRPS-I. After mapping, we found a number of these genes overlapped between groups. These genes we identified are very likely to be expressed in nociceptive sensory neurons and have been previously found to be related with human pain conditions. The rat CPIP model is widely used to mimic human CRPS-I and considered to be a neuropathic pain model. ${ }^{16,39}$ However, it is still not known whether the CPIP model displays any signatures of gene changes similar with other neuropathic models. In order to address this issue, we continued to compare the DEGs of the CPIP model with the rat SNI and SNL models, two well-established rat neuropathic pain models, as well as with CFA-induced inflammatory pain model. We found that the CPIP rat model shares many similar altered genes with two neuropathic pain models but little with the inflammatory pain model, suggesting CPIP model reflects a neuropathic pain-like pathology in primary sensory ganglia.

\section{Conclusion}

Our study presented by far the first study to perform transcriptome analysis of gene changes and key pathways using RNA-Seq in primary sensory ganglia of an animal model of CRPS-I. We identified a number of DEGs and pathways that may be potentially involved in pain mechanisms of CRPS-I. These findings may allow us to gain more mechanistic insights into the pain mechanisms of CRPS-I, which in turn may help to develop more effective approaches for treating pain conditions associated with CRPS-I.

\section{Acknowledgments}

This project is supported by funding from Zhejiang Provincial Natural Science Funds for Distinguished Young Scholars (LR17H270001), the National Natural Science Foundation of China (81873365 and 81603676), Qianjiang Talent Program (QJD1702020), 5151 Talent Program of Zhejiang Chinese Medical University, New Star Talent Program of Zhongshan Hospital of Zhejiang Province (2016xxrc02) to Boyi Liu, and Scientific Research Funding of Zhejiang Chinese Medical University to Boyu Liu (2018ZY19). Contents are solely the responsibility of the authors and do not necessarily represent the views of the funders.

\section{Author contributions}

All authors contributed to data analysis, drafting or revising the article, gave final approval of the version to be published, and agree to be accountable for all aspects of the work.

\section{Disclosure}

The authors report no conflicts of interest in this work.

\section{References}

1. Andersson DA, Gentry C, Moss S, Bevan S. Transient receptor potential A1 is a sensory receptor for multiple products of oxidative stress. $J$ Neurosci. 2008;28(10):2485-2494. doi:10.1523/JNEUROSCI.536907.2008

2. Athie MCP, Vieira AS, Teixeira JM, et al. Transcriptome analysis of dorsal root ganglia's diabetic neuropathy reveals mechanisms involved in pain and regeneration. Life Sci. 2018;205:54-62. doi:10.1016/j. lfs. 2018.05 .016

3. Basbaum AI, Bautista DM, Scherrer G, Julius D. Cellular and molecular mechanisms of pain. Cell. 2009;139(2):267-284. doi:10.1016/j. cell.2009.09.028

4. Berthelot JM. Current management of reflex sympathetic dystrophy syndrome (complex regional pain syndrome type I). Joint Bone Spine. 2006;73(5):495-499. doi:10.1016/j.jbspin.2005.11.022

5. Birklein F, Ajit SK, Goebel A, Perez R, Sommer C. Complex regional pain syndrome - phenotypic characteristics and potential biomarkers. Nat Rev Neurol. 2018;14(5):272-284. doi:10.1038/nrneurol.2018.20

6. Birklein F, Drummond PD, Li W, et al. Activation of cutaneous immune responses in complex regional pain syndrome. J Pain. 2014;15(5):485-495. doi:10.1016/j.jpain.2014.01.490 
7. Borges G, Berrocoso E, Mico JA, Neto F. ERK1/2: function, signaling and implication in pain and pain-related anxio-depressive disorders. Prog Neuropsychopharmacol Biol Psychiatry. 2015;60:77-92. doi:10.1016/j.pnpbp.2015.02.010

8. Caceres AI, Liu B, Jabba SV, Achanta S, Morris JB, Jordt SE. Transient receptor potential cation channel subfamily $\mathrm{M}$ member 8 channels mediate the anti-inflammatory effects of eucalyptol. $\mathrm{Br}$ J Pharmacol. 2017;174:867-879. doi:10.1111/bph.13760

9. Chai W, Tai Y, Shao X, et al. Electroacupuncture alleviates pain responses and inflammation in a rat model of acute gout arthritis. Evid Based Complement Alternat Med. 2018;2018:2598975. doi:10.1155/2018/9567061

10. Chang M, Smith S, Thorpe A, Barratt MJ, Karim F. Evaluation of phenoxybenzamine in the CFA model of pain following gene expression studies and connectivity mapping. Mol Pain. 2010;6:56. doi:10.1186/1744-8069-6-56

11. Chattopadhyay S, Myers RR, Janes J, Shubayev V. Cytokine regulation of MMP-9 in peripheral glia: implications for pathological processes and pain in injured nerve. Brain Behav Immun. 2007;21 (5):561-568. doi:10.1016/j.bbi.2006.10.015

12. Chen W, Ding H, Zhou X, Lin H, Chou KC. iRNA(m6A)-PseDNC: identifying N6-methyladenosine sites using pseudo dinucleotide composition. Anal Biochem. 2018;561-562:59-65. doi:10.1016/j. ab.2018.09.002

13. Chung MK, Park J, Asgar J, Ro JY. Transcriptome analysis of trigeminal ganglia following masseter muscle inflammation in rats. Mol Pain. 2016;12. doi:10.1177/1744806916668526

14. Cobos EJ, Nickerson CA, Gao F, et al. Mechanistic differences in neuropathic pain modalities revealed by correlating behavior with global expression profiling. Cell Rep. 2018;22(5):1301-1312. doi:10.1016/j.celrep.2018.01.006

15. Coderre TJ, Bennett GJ. A hypothesis for the cause of complex regional pain syndrome-type I (reflex sympathetic dystrophy): pain due to deep-tissue microvascular pathology. Pain Med. 2010;11 (8):1224-1238. doi:10.1111/j.1526-4637.2010.00911.x

16. Coderre TJ, Xanthos DN, Francis L, Bennett GJ. Chronic post-ischemia pain (CPIP): a novel animal model of complex regional pain syndrome-type I (CRPS-I; reflex sympathetic dystrophy) produced by prolonged hindpaw ischemia and reperfusion in the rat. Pain. 2004;112(1-2):94-105. doi:10.1016/j.pain.2004.08.001

17. Costigan M, Belfer I, Griffin RS, et al. Multiple chronic pain states are associated with a common amino acid-changing allele in KCNS1. Brain. 2010;133(9):2519-2527. doi:10.1093/brain/awq195

18. Cruz CD, Neto FL, Castro-Lopes J, McMahon SB, Cruz F. Inhibition of ERK phosphorylation decreases nociceptive behaviour in monoarthritic rats. Pain. 2005;116(3):411-419. doi:10.1016/j.pain.2005.05.031

19. De Logu F, Nassini R, Materazzi S, et al. Schwann cell TRPA1 mediates neuroinflammation that sustains macrophage-dependent neuropathic pain in mice. Nat Commun. 2017;8(1):1887. doi:10.1038/s41467-017-01739-2

20. Dev R, Srivastava PK, Iyer JP, Dastidar SG, Ray A. Therapeutic potential of matrix metalloprotease inhibitors in neuropathic pain. Expert Opin Investig Drugs. 2010;19(4):455-468. doi:10.1517/ 13543781003643486

21. Du H, Shi J, Wang M, An S, Guo X, Wang Z. Analyses of gene expression profiles in the rat dorsal horn of the spinal cord using RNA sequencing in chronic constriction injury rats. $J$ Neuroinflammation. 2018;15(1):280. doi:10.1186/s12974-018-1220-7

22. Flegel C, Schobel N, Altmuller J, et al. RNA-Seq analysis of human trigeminal and dorsal root ganglia with a focus on chemoreceptors PLoS One. 2015;10(6):e0128951. doi:10.1371/journal.pone.0128951

23. Franceschini A, Szklarczyk D, Frankild S, et al. STRING v9.1: protein-protein interaction networks, with increased coverage and integration. Nucleic Acids Res. 2013;41(Databaseissue):D808-D815. doi:10.1093/nar/gks 1094
24. Goswami SC, Mishra SK, Maric D, et al. Molecular signatures of mouse TRPV1-lineage neurons revealed by RNA-Seq transcriptome analysis. $J$ Pain. 2014;15(12):1338-1359. doi:10.1016/j. jpain.2014.09.010

25. Grothusen JR, Alexander G, Erwin K, Schwartzman R. Thermal pain in complex regional pain syndrome type I. Pain Physician. 2014;17 (1):71-79.

26. Guptarak J, Wanchoo S, Durham-Lee J, et al. Inhibition of IL-6 signaling: a novel therapeutic approach to treating spinal cord injury pain. Pain. 2013;154(7):1115-1128. doi:10.1016/j.pain.2013.03.026

27. Hord ED, Oaklander AL. Complex regional pain syndrome: a review of evidence-supported treatment options. Curr Pain Headache Rep. 2003;7(3):188-196.

28. $\mathrm{Hu} \mathrm{G}$, Huang $\mathrm{K}, \mathrm{Hu} \mathrm{Y}$, et al. Single-cell RNA-seq reveals distinct injury responses in different types of DRG sensory neurons. Sci Rep. 2016;6:31851. doi:10.1038/srep31851

29. Imai S, Ikegami D, Yamashita A, et al. Epigenetic transcriptional activation of monocyte chemotactic protein 3 contributes to long-lasting neuropathic pain. Brain. 2013;136(Pt 3):828-843. doi:10.1093/brain/aws330

30. Jiang BC, Cao DL, Zhang X, et al. CXCL13 drives spinal astrocyte activation and neuropathic pain via CXCR5. J Clin Invest. 2016;126 (2):745-761. doi:10.1172/JCI81950

31. Jiang BC, Sun WX, He LN, Cao DL, Zhang ZJ, Gao YJ. Identification of IncRNA expression profile in the spinal cord of mice following spinal nerve ligation-induced neuropathic pain. $\mathrm{Mol}$ Pain. 2015;11:43. doi:10.1186/s12990-015-0047-9

32. Jin EH, Zhang E, Ko Y, et al. Genome-wide expression profiling of complex regional pain syndrome. PLoS One. 2013;8(11):e79435. doi:10.1371/journal.pone.0079435

33. Kawasaki Y, Xu ZZ, Wang X, et al. Distinct roles of matrix metalloproteases in the early- and late-phase development of neuropathic pain. Nat Med. 2008;14(3):331-336. doi:10.1038/nm1723

34. Kiguchi N, Maeda T, Kobayashi Y, Kondo T, Ozaki M, Kishioka $S$. The critical role of invading peripheral macrophage-derived interleukin- 6 in vincristine-induced mechanical allodynia in mice. Eur J Pharmacol. 2008;592(1-3):87-92. doi:10.1016/j.ejphar.2008.07.008

35. Klafke JZ, da Silva MA, Rossato MF, et al. Acute and chronic nociceptive phases observed in a rat hind paw ischemia/reperfusion model depend on different mechanisms. Pflugers Arch. 2016;468 (2):229-241. doi:10.1007/s00424-015-1746-9

36. LaCroix-Fralish ML, Austin JS, Zheng FY, Levitin DJ, Mogil JS. Patterns of pain: meta-analysis of microarray studies of pain. Pain. 2011;152(8):1888-1898. doi:10.1016/j.pain.2011.04.014

37. Liu B, Tai Y, Caceres AI, et al. Oxidized phospholipid OxPAPC activates TRPA1 and contributes to chronic inflammatory pain in mice. PLoS One. 2016;11(11):e0165200. doi:10.1371/journal. pone. 0165200

38. Livak KJ, Schmittgen TD. Analysis of relative gene expression data using real-time quantitative PCR and the 2(-Delta Delta $\mathrm{C}(\mathrm{T})$ ) method. Methods. 2001;25(4):402-408. doi:10.1006/meth.2001.1262

39. Luo X, Tai WL, Sun L, et al. Crosstalk between astrocytic CXCL12 and microglial CXCR4 contributes to the development of neuropathic pain. Mol Pain. 2016;12.

40. Mishra SK, Tisel SM, Orestes P, Bhangoo SK, Hoon MA. TRPV1lineage neurons are required for thermal sensation. EMBO J. 2011;30 (3):582-593. doi:10.1038/emboj.2010.325

41. Murakami T, Kanchiku T, Suzuki H, et al. Anti-interleukin-6 receptor antibody reduces neuropathic pain following spinal cord injury in mice. Exp Ther Med. 2013;6(5):1194-1198. doi:10.3892/ etm.2013.1296

42. Rasmussen VF, Karlsson P, Drummond PD, et al. Bilaterally reduced intraepidermal nerve fiber density in unilateral CRPS-I. Pain Med. 2018;19(10):2021-2030. doi:10.1093/pm/pnx240 
43. Reimer M, Rempe T, Diedrichs C, Baron R, Gierthmuhlen J. Sensitization of the nociceptive system in complex regional pain syndrome. PLoS One. 2016;11(5):e0154553. doi:10.1371/journal.pone.0154553

44. Shannon P, Markiel A, Ozier O, et al. Cytoscape: a software environment for integrated models of biomolecular interaction networks. Genome Res. 2003;13(11):2498-2504. doi:10.1101/gr.1239303

45. Su TF, Zhao YQ, Zhang LH, et al. Electroacupuncture reduces the expression of proinflammatory cytokines in inflamed skin tissues through activation of cannabinoid CB2 receptors. Eur J Pain. 2012;16(5):624-635. doi:10.1002/j.1532-2149.2011.00055.x

46. Tang Y, Liu L, Xu D, et al. Interaction between astrocytic colony stimulating factor and its receptor on microglia mediates central sensitization and behavioral hypersensitivity in chronic post ischemic pain model. Brain Behav Immun. 2018;68:248-260. doi:10.1016/j. bbi.2017.10.023

47. Terkelsen AJ, Gierthmuhlen J, Finnerup NB, Hojlund AP, Jensen TS. Bilateral hypersensitivity to capsaicin, thermal, and mechanical stimuli in unilateral complex regional pain syndrome. Anesthesiology. 2014;120(5):1225-1236. doi:10.1097/ALN.0000000000000220

48. Thakur M, Crow M, Richards N, et al. Defining the nociceptor transcriptome. Front Mol Neurosci. 2014;7:87. doi:10.3389/ fnmol.2014.00087
49. Urits I, Shen AH, Jones MR, Viswanath O, Kaye AD. Complex regional pain syndrome, current concepts and treatment options. Curr Pain Headache Rep. 2018;22(2):10. doi:10.1007/s11916-0180667-7

50. Wu XB, Cao DL, Zhang X, et al. CXCL13/CXCR5 enhances sodium channel Nav1.8 current density via p38 MAP kinase in primary sensory neurons following inflammatory pain. Sci Rep. 2016;6:34836. doi:10.1038/srep34836

51. Xing F, Kong C, Bai L, et al. CXCL12/CXCR4 signaling mediated ERK1/2 activation in spinal cord contributes to the pathogenesis of postsurgical pain in rats. Mol Pain. 2017;13:1744806917718753. doi:10.1177/1744806917718753

52. Zhang H, Li Y, de Carvalho-Barbosa M, et al. Dorsal root ganglion infiltration by macrophages contributes to paclitaxel chemotherapy-induced peripheral neuropathy. J Pain. 2016;17 (7):775-786. doi:10.1016/j.jpain.2016.02.011

53. Zhang ZJ, Jiang BC, Gao YJ. Chemokines in neuron-glial cell interaction and pathogenesis of neuropathic pain. Cell Mol Life Sci. 2017;74(18):3275-3291. doi:10.1007/s00018-017-2513-1

54. Zhou YQ, Liu Z, Liu ZH, et al. Interleukin-6: an emerging regulator of pathological pain. $J$ Neuroinflammation. 2016;13(1):141. doi:10.1186/s12974-016-0607-6

\section{Publish your work in this journal}

The Journal of Pain Research is an international, peer reviewed, open access, online journal that welcomes laboratory and clinical findings in the fields of pain research and the prevention and management of pain. Original research, reviews, symposium reports, hypothesis formation and commentaries are all considered for publication. The manuscript management system is completely online and includes a very quick and fair peer-review system, which is all easy to use. Visit http:// www.dovepress.com/testimonials.php to read real quotes from published authors. 